\title{
Myocardial blood flow quantification conventional single photon tracers: Yet another critical appraisal
}

\section{Atsutaka Okizaki et al: Noninvasive estimation of quantitative myocardial blood flow with Tc-99m MIBI by a compartment model analysis in rat}

\author{
Stephan G. Nekolla, Ph.D., FESC, ${ }^{a}$ and Christoph Rischpler, MD ${ }^{b}$ \\ a Nuklearmedizinische Klinik und Poliklinik, Klinikum rechts der Isar, Technische Universität \\ München, Munich, Germany \\ b Nuklearmedizinische Klinik und Poliklinik, Universitätsklinikum Essen, Essen, Germany
}

Received Oct 23, 2018; accepted Oct 23, 2018

doi: $10.1007 / \mathrm{s} 12350-018-01509-7$

\section{See related article, pp. 1368-1374}

Almost $* 4$ years ago, we published an editorial on a similar topic, namely the absolute quantification of myocardial blood flow (MBF) using conventional SPECT tracers and cameras. ${ }^{2,3}$ Now, this editorial references a publication where some core elements are changed and some are similar: the targets are rodents instead of humans, dynamic planar instead to tomographic imaging is used, the kinetic modeling is used but the SPECT system follows a conventional design (although preclinical). Still, the tracer is a conventional one (even-but for well-known reasons-the injected doses are similar). In 2014, we summarized the following:

So, what is the immediate message one can derive from the paper by Klein et al. ? On the negative side, it is a confirmation that conventional SPECT agents are no ideal candidates for kinetic modeling
Reprint requests: Stephan G. Nekolla, Ph.D., FESC, Nuklearmedizinische Klinik und Poliklinik, Klinikum rechts der Isar, Technische Universität München, Ismaninger Str. 22, 81675 Munich, Germany; stephan.nekolla@tum.de

J Nucl Cardiol 2020;27:1375-7.

$1071-3581 / \$ 34.00$

Copyright (c) 2018 American Society of Nuclear Cardiology. and thus quantification of myocardial blood flow and flow reserve in absolute terms. On the positive side, however, the authors show that even conventional cameras with suitable collimators, wellvalidated attenuation and scatter correction and optimized image reconstruction capabilities have the potential to acquire dynamic data which-at least-are the prerequisites to become truly quantitative. Now, throw in a suitable SPECT perfusion tracer, and nuclear cardiology is good to go another two decades_-at least!

Unfortunately, most of these conclusions still are true-especially on the tracer side, no real advances happened in the last four years. Interestingly, also the hopes back then that a new PET agent $\left({ }^{18} \mathrm{~F}\right.$ Flurpiridaz) could be widely available by now, did not happen. To some extent, a valid conclusion might be that the use of already approved radiopharmaceuticals and the development of new methods for them is still worthwhile as the regulatory hurdles for new agents will not decrease.

Thus-even in light of challenges when it comes to $\mathrm{Mo} / \mathrm{Tc}$ supply - the optimized logistical advantage of single photon imaging is fascinating. If it comes to side effects in patients, a pragmatic view sees quite some

\footnotetext{
* As in an earlier editorial, we took the words "critical appraisal" from a review by Wolfgang A. Weber on the use of $\mathrm{PET} / \mathrm{MR}$ on oncological imaging as we see still some parallel aspects $\left({ }^{1}\right.$ Weber WA. PET/MR Imaging: A Critical Appraisal. Journal of Nuclear Medicine: Official Publication, Society of Nuclear Medicine 2014;55:56S-58S.)
} 
advantages over iodinated contrast agents or gadolinium chelates. That the quantification of MBF and coronary flow reserve (CFR) is of significant clinical interest might be simply derived from the fact that its assessment (or variants of them) directly within the cath $1 a b^{4,5}$ or their estimation based on CT imaging using computational methods ${ }^{6}$ is ever increasing.

Thus, blood flow quantification with SPECT would still be a very welcomed addition to the armamentarium of nuclear cardiology both in general, and as shown in their paper, in preclinical research. Dynamic imaging with SPECT is challenging both for humans and rodents, although much progress was observed in the clinical area with conventional photo-multiplier based SPECT systems ${ }^{7,8,9}$ and solid-state photon detectors. ${ }^{10}$ Okizaki and colleagues addressed the problem with a novel (sic!) idea, namely dynamic planar imaging and time-to-peak maps for an optimized approach to define tissue and blood regions-of-interest. From this, the kinetic data are retrieved and kinetic modeling is used to derive MBF. In general, compartmental or kinetic modeling splits the myocardium into two or more compartments (e.g., blood and tissue) and describes the exchange between the two with rate constants. In the end, this results in differential equations which can be numerically solved. Then, the fitting of the measured data against the model provides the desired exchange constants. As usual in computational methods, the higher the noise in the data, the higher the variability of this assessment. The same logic applies for the correction of the rate constant from blood to tissue: is the extraction fraction high (such as for ${ }^{18} \mathrm{NH}_{3}$ PET), only modest corrections (i.e., amplifications) are needed: if it is low, the required (strong) amplification) for higher flows amplifies noise. Unfortunately, the typical SPECT agent shows a rather lower extraction: introduced $2-3$ decades ago, ${ }^{99 \mathrm{~m}} \mathrm{Tc}$ labeled compounds such as sestamibi and tetrofosmin demonstrate a so-called 'roll-off', phenomenon in their extraction: basically, at higher flows the tracer is not as rapidly extracted from the blood stream due to limitations when crossing membranes etc. as under low flow conditions. ${ }^{11,12}$ In static imaging this does not really matter in daily routine, although it would be highly interesting: with a tracer retention directly proportional to the MBF, one could calculate a SUV (standard uptake value, i.e., tracer uptake normalized with patient weight and injected dose) based flow reserve using pharmaceutical stress. This concept, however works potentially only for tracers with very high extraction ${ }^{13}$ - but points to the complexity of the matter.

Okizaki et al. confirm the respective limitation of MIBI in their study, the pattern of the correlation of microsphere derived and imaging based MBF is, however, somewhat unusual and differs from previous data in larger animals or humans for higher flows. Most likely, this reflects a well-known finding in small animals that the input function is harder to resolve because of the spatial dimensions and the effects are more pronounced at higher flows. Another factor influencing this is the source of data, namely dynamic planar imaging, and the lack of scatter and attenuation correction. Finally, the authors used Dr. Leppo's correction derived in 1989 for rats with quite different hardware. ${ }^{11}$ Interestingly, there would be more recent curves available, e.g., in a porcine model. ${ }^{14}$ This raises, however, an interesting question: which correction function should be actually used ? Especially in the context of low extraction tracers such as MIBI, tetrofosmin, or rubidium, this has tremendous consequences for the reproducibility. This might serve as an example: a recent study compared several MBF quantification software solutions for Rubidium-82 MBF estimation. ${ }^{15}$ A group of packages used a correction derived from sequential examinations in humans with ${ }^{13} \mathrm{NH}_{3}$ and ${ }^{82} \mathrm{Rb}^{16}$ and showed in a statistical analysis the highest degree of agreement. In contrast, the agreement between the aforementioned approach and a conventionally derived model based on simultaneous microspheres measurements in animal models did not correlate to this degree, ${ }^{17}$ as the extraction fraction correction curves differed: not that much, but given the significant amplification it mattered. The definitive lesson learned is that software needs to be tested for exchangeabilitywell-known in nuclear cardiology from the quantification of global functional values ${ }^{18}$ - but enhanced for MBF quantification, as ground truth is increasingly hard to find.

However, is the search for ideal solutions something which matches clinical realities? The extremely wide spread use of FDG in oncological PET might serve as an example. It is far from perfect but works well if the user is aware of its limitations. And there is another analogy: with the ever increasing capabilities of PET scanners, an old idea might become a reality-dynamic whole body PET to improve from semi-quantitative values (SUV) to rather absolute kinetic constants. Or, is high extraction really always needed ? An example is MBF quantification with MRI: although these contrast agents are not extracted by the cell at all, sophisticated modeling (and sophisticated should be spelled in capital letters) show that it is in principle feasible $\{$ Kunze, 2018, \#67\}.

So, four years later, let us raise the same question: what is the immediate message one can derive from the paper by Okizaki et al.? Again, conventional SPECT agents show only a modest extraction and are no ideal candidates for quantification of myocardial blood flow and flow reserve in absolute terms. However, as discussed above, looking for ideal solutions is something 
which might be not always simply aligned with clinical realities.

Thus, never stop exploring, but keep an eye on Occam's razor.

\section{Disclosure}

None.

\section{References}

1. Weber WA. PET/MR imaging: A critical appraisal. J Nucl Med. 2014;55:56S-8S.

2. Klein R, Hung GU, Wu TC, et al. Feasibility and operator variability of myocardial blood flow and reserve measurements with (9)(9)mTc-sestamibi quantitative dynamic SPECT/CT imaging. J Nucl Cardiol. 2014;21:1075-88.

3. Nekolla SG, Rischpler C, Nakajima K. Myocardial blood flow quantification with SPECT and conventional tracers: a critical appraisal. J Nucl Cardiol. 2014;21:1089-91.

4. Tonino PA, De Bruyne B, Pijls NH, et al. Fractional flow reserve versus angiography for guiding percutaneous coronary intervention. N Engl J Med. 2009;360:213-24.

5. Fearon WF, Shilane D, Pijls NH, et al. Cost-effectiveness of percutaneous coronary intervention in patients with stable coronary artery disease and abnormal fractional flow reserve. Circulation. 2013;128:1335-40.

6. Pontone G, Baggiano A, Andreini D, et al. Stress computed tomography perfusion versus fractional flow reserve $\mathrm{CT}$ derived in suspected coronary artery disease: The PERFECTION study. JACC Cardiovascular Imaging. 2018. https://doi.org/10.1016/j.jc mg.2018.08.023.

7. Nakajima K, Taki J, Bunko H, et al. Dynamic acquisition with a three-headed SPECT system: Application to technetium 99mSQ30217 myocardial imaging. J Nucl Med. 1991;32:1273-7.

8. Smith AM, Gullberg GT, Christian PE, Datz FL. Kinetic modeling of teboroxime using dynamic SPECT imaging of a canine model. $\mathrm{J}$ Nucl Med. 1994;35:484-95.
9. Khare HS, Dibella EV, Kadrmas DJ, Christian PE, Gullberg GT. Comparison of static and dynamic cardiac perfusion thallium-201 SPECT. IEEE Trans Nucl Sci. 2001;48:774.

10. Ben-Haim S, Murthy VL, Breault C, et al. Quantification of myocardial perfusion reserve using dynamic SPECT imaging in humans: A feasibility study. J Nucl Med. 2013;54:873-9.

11. Leppo JA, Meerdink DJ. Comparison of the myocardial uptake of a technetium-labeled isonitrile analogue and thallium. Circ Res. 1989;65:632-9.

12. Glover DK, Ruiz M, Yang JY, Smith WH, Watson DD, Beller GA. Myocardial 99mTc-tetrofosmin uptake during adenosine-induced vasodilatation with either a critical or mild coronary stenosis: comparison with $201 \mathrm{Tl}$ and regional myocardial blood flow. Circulation. 1997;96:2332-8.

13. Sherif HM, Nekolla SG, Saraste A, et al. Simplified quantification of myocardial flow reserve with flurpiridaz F 18: validation with microspheres in a pig model. J Nucl Med. 2011;52:617-24.

14. Wells RG, Timmins R, Klein R, et al. Dynamic SPECT measurement of absolute myocardial blood flow in a porcine model. $\mathrm{J}$ Nucl Med. 2014;55:1685-91.

15. Nesterov SV, Deshayes E, Sciagra R, et al. Quantification of myocardial blood flow in absolute terms using (82)Rb PET imaging: The RUBY-10 study. JACC Cardiovasc Imaging. 2014;7:1119-27.

16. Lortie M, Beanlands RS, Yoshinaga K, Klein R, Dasilva JN, DeKemp RA. Quantification of myocardial blood flow with 82Rb dynamic PET imaging. Eur J Nucl Med Mol Imaging. 2007;34:1765-74.

17. Lautamaki R, George RT, Kitagawa K, et al. Rubidium-82 PET$\mathrm{CT}$ for quantitative assessment of myocardial blood flow: Validation in a canine model of coronary artery stenosis. Eur J Nucl Med Mol Imaging. 2009;36:576-86.

18. Stegger L, Lipke CS, Kies P, et al. Quantification of left ventricular volumes and ejection fraction from gated $99 \mathrm{mTc}$-MIBI SPECT: validation of an elastic surface model approach in comparison to cardiac magnetic resonance imaging, 4D-MSPECT and QGS. Eur J Nucl Med Mol Imaging. 2007;34:900-9. 